\title{
Identification of Candidate Genes Involved in Renal Ischemia/Reperfusion Injury
}

\author{
Ming Su, ${ }^{1}$ Xinyi Hu, ${ }^{2}$ Jun Lin, ${ }^{2}$ Lei Zhang, ${ }^{2}$ Wen Sun, ${ }^{2}$ Jian Zhang, ${ }^{2}$ Ye Tian, ${ }^{2}$ and Wei Qiu ${ }^{2}$
}

Renal ischemia/reperfusion injury (IRI) is a main risk factor for the occurrence of delayed graft function or primary graft nonfunction of kidney transplantation. However, it lacks ideal molecular markers for indicating IRI in kidney transplantation. The present study is to explore novel candidate genes involved in renal IRI. Experimental renal IRI mouse models were constructed, and the differentially expressed genes were screened using a microarray assay. Gene Ontology and Kyoto Encyclopedia of Genes and Genomes enrichment analysis were performed. The expression of genes was detected using real-time qPCR assay. Western blotting and immunohistochemistry staining assays were performed for protein determination. We identified that renal IRI induced the upregulation of SPRR $2 F, S P R R 1 A, M M P-10$, and long noncoding RNA (lncRNA) Malatl in kidney tissues for 479.3-, 4.98-, 238.1-, and 3.79-fold, respectively. The expression of miR-139-5p in kidney tissues of IRI-treated mice was decreased to $40.4 \%$ compared with the sham-operated mice. These genes are associated with keratinocyte differentiation, regeneration and repair of kidney tissues, extracellular matrix degradation and remodeling, inflammation, and cell proliferation in renal IRI. Identification of novel biomarkers involved in renal IRI may provide evidences for the diagnosis and treatment of renal IRI.

Keywords: ischemia/reperfusion injury, kidney transplantation, SPRR2F, MMP-10, miR-139-5p

\section{Introduction}

$\mathbf{K}$ IDNEY TRANSPLANTATION IS an effective therapy for most of the end-stage renal disease patients. As the extending criteria of deceased kidney donors, the rates of delayed graft function (DGF) or primary graft nonfunction (PGN) are increasing. The occurrence of DGF and PGN can be caused by many aspects, such as ischemia/reperfusion injury (IRI), immunological rejection, primary diseases of the recipients, and the quality of the graft. However, there is no effective treatment for DGF and PGN after kidney transplantation.

IRI is one of the most important but unavoidable risk factors for causing the impairment of graft function. Renal IRI triggers the generation of reactivate oxygen species, causing oxidative injury, acute inflammation and eventually necrosis, and apoptosis of renal tubular epithelial cells (Gu et al., 2018; Situmorang and Sheerin, 2018). These responses can evoke the anti-inflammatory and repair processes to initiate the recovery of the graft function (SanchezNino et al., 2016; Pressly and Park, 2017). On the contrary, many factors released during the IRI, such as transforming growth factor- $\beta$, matrix metalloproteinases (MMPs), and altered extracellular matrix (ECM) components, can induce epithelial-mesenchymal transition, ECM remodeling, and interstitial fibrosis (Jain et al., 2000; Danobeitia et al., 2017; Kim et al., 2017; Liu et al., 2018), which hinder the recovery of complete function. Therefore, IRI is a complex and multistep pathological process that the mechanism has not been fully clarified yet. Identifying novel targets of renal IRI is crucial for exploring the diagnosis and treatment of DGF or PGN after kidney transplantation. In the present study, we screened for differentially expressed genes in renal IRI based on an experimental IRI model.

\section{Materials and Methods}

\section{Animal studies}

All the studies related to animals were approved by the Beijing Friendship Hospital Animal Care and Use Committee (approval no.: 2018-P2-167-01). The renal IRI experiments were performed as previously described (Hesketh

\footnotetext{
${ }^{1}$ Department of Clinical Laboratory, Peking University People's Hospital, Beijing, People's Republic of China.

${ }^{2}$ Department of Urology, Beijing Friendship Hospital, Capital Medical University, Beijing, People's Republic of China.
}

(C) Ming Su et al., 2019; Published by Mary Ann Liebert, Inc. This Open Access article is distributed under the terms of the Creative Commons Attribution Noncommercial License (http://creativecommons.org/licenses/by-nc/4.0/) which permits any noncommercial use, distribution, and reproduction in any medium, provided the original author(s) and the source are cited. 
et al., 2014). In brief, 8-week-old male C57/BL6 mice were anesthetized through intraperitoneal injection of pentobarbital sodium $(0.05 \mathrm{mg} / \mathrm{g}$ body weight). The mice were randomly divided into injury and sham-treated groups. For IRI treatment, the mice were subjected to $45 \mathrm{~min}$ of ischemia by bilaterally clamping the renal artery. After $24 \mathrm{~h}$ of reperfusion, the mice were euthanized and the kidney samples were immediately frozen at $-80^{\circ} \mathrm{C}$ or fixed in $4 \%$ paraformaldehyde for further analysis.

\section{Microarray assay}

Total RNA from the kidney samples was extracted using TRIzol reagent (Thermo Fisher Scientific, Schuylerville, NY). The differentially expressed genes were detected using an Affymetrix Mouse Genome 4302.0 microarray (Thermo Fisher Scientific) from three mice of renal IRI and three sham-treated mice. The experimental procedures for microarray were performed at the Shanghai Biotechnology Corporation (Shanghai, China). The differential expression genes fulfill at least twofold change and $p<0.05$ were considered for further analysis. Gene Ontology (GO) and Kyoto Encyclopedia of Genes and Genomes (KEGG) enrichment analysis were performed.

RNA extraction and real-time $\mathrm{QPCR}$ analysis. Total RNA from kidney samples was extracted with the miRNeasy Mini Kit (Qiagen, Hilden, Germany). For quantitative detection of mRNA or lncRNA, the complementary DNAs (cDNAs) were synthesized with a cDNA Synthesis Kit (Takara, Dalian, China). The real-time qPCR was performed by using the following specific primers: $M M P-10,5^{\prime}$-TCCA GGAATTGAGCCACAAG-3' and 5'-GCATTGGGGTCAA ACTCGAAC-3'; SPRRIA, 5'-GAGAACCTGCTCTTCTC TGAGT-3' and $5^{\prime}$-CAAGGTTCCTGGGGTGGTG-3'; SPR $R 2 F, 5^{\prime}$-GGAGAACCTGATCCTGAGGC-3' and $5^{\prime}$-CCCT GTCCTGAAGACTGCTG-3'; Malat 1, 5'-TGCAGTGTGC CAATGTTTCG-3' and 5'-GGCCAGCTGCAAACATTCAA$3^{\prime}$. GAPDH was used as an internal control, and the primers are: 5'-GGCAAATTCAACGGCACAGT-3' and 5'-AGAT GGTGATGGGCTTCCC-3'. For the detection of mature form of miRNA-139-5p, the specific primer containing a stem-loop structure (5'-CTCAACTGGTGTCGTGGAGTCGGCAATTC AGTTGAGACTGGAGA-3') was used for reverse transcription, and the specific primers were used for amplification: 5'ACACTCCAGCTGGGTCTACAGTGCACGTGTC- $3^{\prime}$ and 5'-TGGTGTCGTGGAGTCG-3'. The ribosomal U6 was used as an internal control for normalization: 5'-CTCGCTTCG GCAGCACA-3' and 5'-AACGCTTCACGAATTTGCGT-3'.

\section{Immunochemistry staining}

Kidney tissues fixed in $4 \%$ paraformaldehyde were dehydrated and embedded in the paraffin. The samples were cut into $4 \mu \mathrm{m}$ thick sections. The sections were then dewaxed and rehydrated. The antigen was repaired by a heating process, and the endogenous peroxidases were blocked with $3 \% \mathrm{H}_{2} \mathrm{O}_{2}$ for $20 \mathrm{~min}$ at room temperature. After washed with phosphate-buffered saline (PBS) for three times, the sections were incubated with rabbit anti-MMP-10 primary antibody (BBI; Sangon Biotech, Shanghai, China) at $4{ }^{\circ} \mathrm{C}$ overnight. After that, the sections were washed three times with PBS and incubated in an HRP/Fab polymer- conjugated secondary antibody (ZSGB-BIO, Beijing, China) for $30 \mathrm{~min}$ at room temperature. The sections were then washed for five times with PBS and were developed using a diaminobenzidine reagent. The nuclei were counter stained with hematoxylin and visualized with a microscopy.

\section{Protein extraction and western blotting}

Total protein from tissue samples were extracted with RIPA lysis buffer containing protease and phosphatase inhibitors. The lysates were centrifuged with 12,000 round per minute at $4^{\circ} \mathrm{C}$ for $10 \mathrm{~min}$. The supernatants were collected and quantified using bicinchoninic acid (BCA) assay (Thermo Fisher Scientific). The protein samples were normalized and diluted at a final concentration of $5 \mu \mathrm{g} / \mu \mathrm{L}$. For electrophoretic separation, a total of $50 \mu \mathrm{g}$ proteins from each sample were loaded onto a sodium dodecyl sulphate (SDS) polyacrylamide gel. After the electrophoresis, the proteins on the SDS polyacrylamide gel were blotted onto a nitrocellulose membrane and then blocked with 5\% nonfat milk at room temperature for $1 \mathrm{~h}$. After that, the membrane was incubated with specific antibodies: rabbit anti-MMP-10 (BBI; Sangon Biotech) and mouse anti- $\beta$-actin (Santa Cruz, Dallas, TX). The membrane was washed with TBS-T and incubated with HRP-conjugated goat anti-rabbit or mouse secondary antibodies (ZSGB-BIO) for $1 \mathrm{~h}$ at room temperature. After washing with TBS-T for four times, the specific bands were developed using Super Signal West Femto Maximum Sensitivity Substrate (Pierce, Rockford, IL) and scanned using Quantity One software V 4.6.2 (Bio-Rad, Hercules, CA). The expression of $\beta$-actin was used as an internal control.

\section{Statistical analysis}

All the statistical calculations were performed with SPSS 19.0 software (SPSS, Inc., Chicago, IL). A two-tailed Student's $t$-test was used for analyzing the differences between two groups. $p<0.05$ was considered as statistically significant.

\section{Results}

\section{Screening for differential expression genes in renal IRI}

Previously, we found 2218 differentially expressed genes, including 1103 upregulated genes and 1115 downregulated genes by using microarray assay (Fig. 1a; Hu et al., 2019). In the present study, GO and KEGG enrichment analyses were performed, and the top 30 enrichments were listed, respectively (Fig. 1b, c, complete data see Supplementary Tables S1 and S2). We found that many essential pathways were associated with renal IRI, such as tumor necrosis factor signaling pathway, MAPK signaling pathway, Hif-1 signaling pathway, ECM-receptor interaction, focal adhesion, keratinocyte development, Toll-like receptor signaling, I $\kappa \mathrm{B} / \mathrm{NF}-\kappa \mathrm{B}$ signaling pathway, positive regulation of pri-miRNA transcription, and metanephric nephron tubule development.

\section{SPRR2F and SPRR1A is upregulated in renal IRI}

Keratinocytes are known to participate in IRI, and the keratinocyte-derived chemokine is a known biomarker for indicating early renal injury before the increase of serum 
a

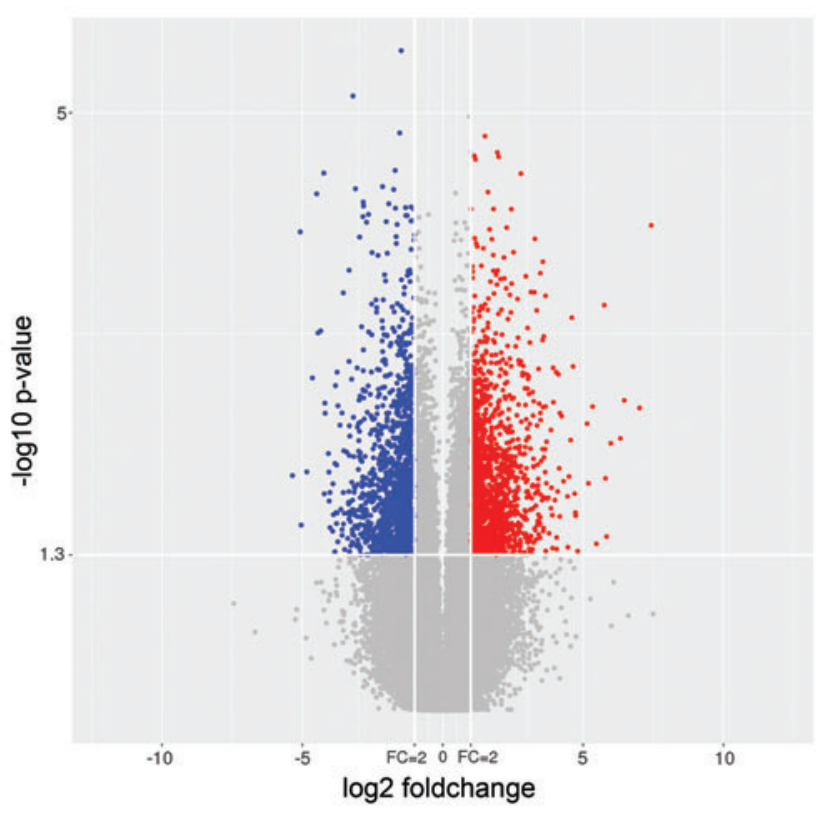

b

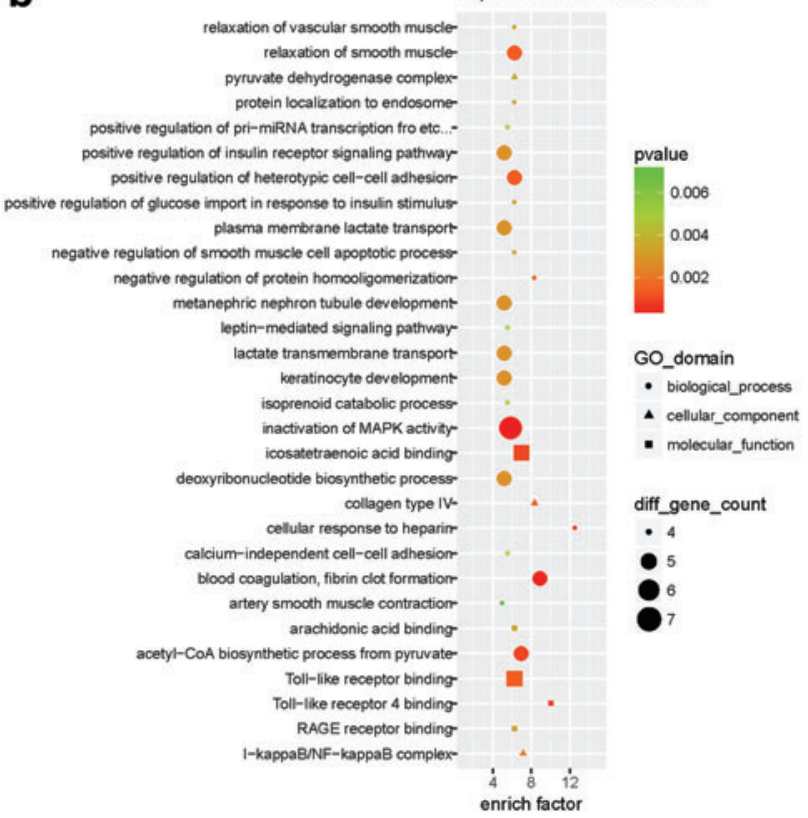

C

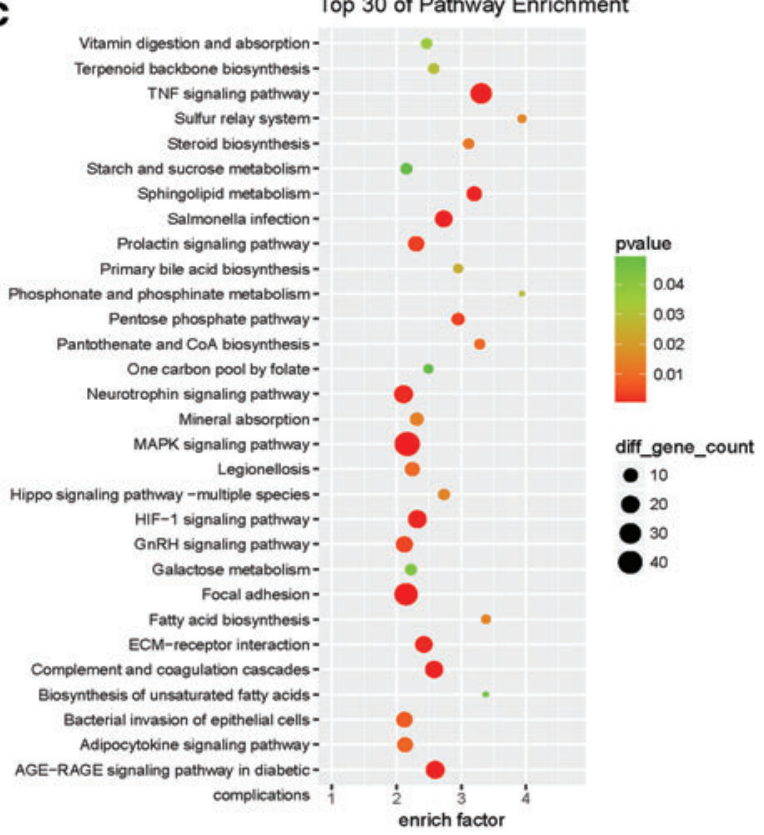

FIG. 1. Screening for differentially expressed genes in renal IRI. (a) Volcano plot diagram of differentially expressed genes ( $\geq 2$-fold, $p<0.05$ ) from IRI-treated and sham-treated mice ( $n=3$ per group). (b, c) Top 30 pathways from Gene Ontology (b) and Kyoto Encyclopedia of Genes and Genomes (c) enrichment analysis are shown. IRI, ischemia/reperfusion injury. The image can be magnified online for greater readability. Color images are available online.

creatinine (Molls et al., 2006). Data from GO enrichment analysis (Fig. 1b) also supported that genes-related keratinocytes might be potential biomarkers of IRI. By analyzing these differentially expressed genes, we found that the expression of $S P R R 2 F$ and SPRRIA, annotated as "keratinocytes differentiation," was significantly upregulated in the kidney tissues of IRI mice. The results from real-time qPCR consistently verified that the expression of $S P R R 2 F$ in kidneys of IRI mice was 479.3-fold higher than the shamoperated mice (Fig. 2a). Likewise, the expression of SPRR1A increased up to 4.98-fold of the sham-treated group
(Fig. 2b). Our data indicated that the $S P R R 2 F$ and $S P R R 1 A$ might be involved in IRI.

\section{The expression of MMP-10 is activated in renal IRI}

ECM degradation and renal remodeling are important pathological changes of IRI hindering the recovery of kidney. During the acute phase, breakdown of normal structures of ECM aggravates kidney injury, whereas the interstitial fibrosis impairs kidney function in the chronic phase. From the data of the differentially expressed genes of IRI, we found 

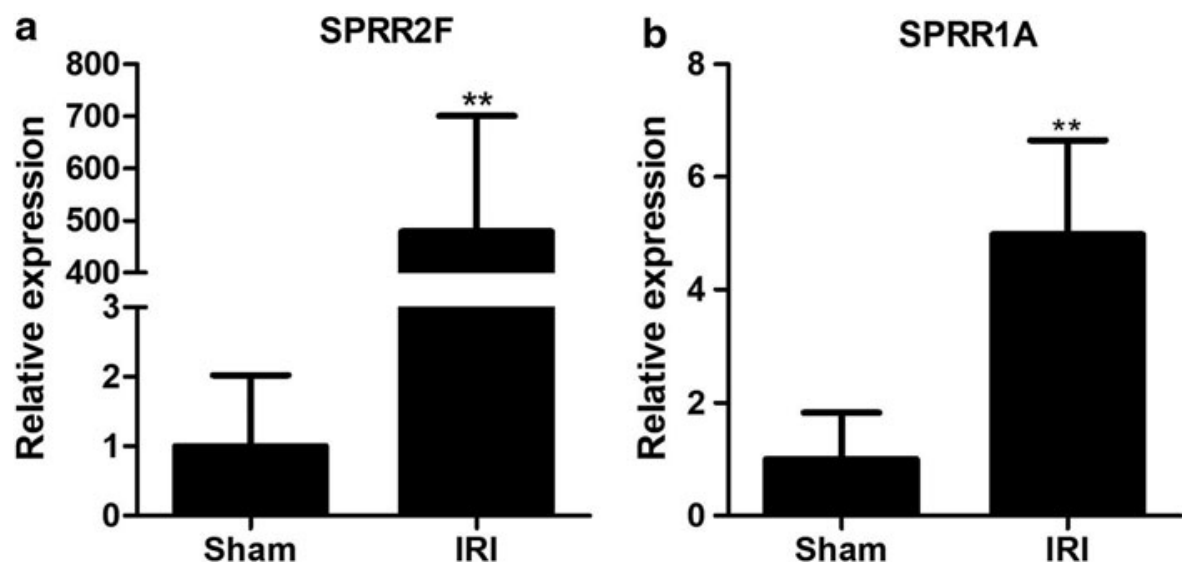

FIG. 2. $S P R R 2 F$ and $S P R R 1 A$ are upregulated in IRI. (a, b) The expression of $S P R R 2 F$ (a) and SPRRIA (b) was determined using a real-time qPCR assay. GAPDH was used as an internal control for normalizing. Data are expressed as mean \pm standard deviation, and $* * p<0.01$ versus the shamoperated group ( $n=4$ per group).

that $M M P-10$, an enzyme mediating the degradation of ECM, was significantly upregulated by IRI treatment. By using realtime qPCR, we observed that $M M P-10$ was highly expressed in all the mice undergone IRI operation (Fig. 3a). In contrast, $M M P-10$ is undetectable in most of the mice of the shamoperated group. The products from real-time qPCR were visualized through an ultraviolet imager (Fig. 3b).
We then analyzed the expression of $M M P-10$ at protein levels, using western blotting. We found that $M M P-10$ was indeed upregulated in kidneys of IRI-treated mice (Fig. 3c, d). Interestingly, $M M P-10$ was presented in kidney tissues of sham-operated mice, which is not paralleled with the results of its mRNA level. Consistently, data from immunohistochemistry staining indicated that $M M P-10$ was a

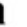

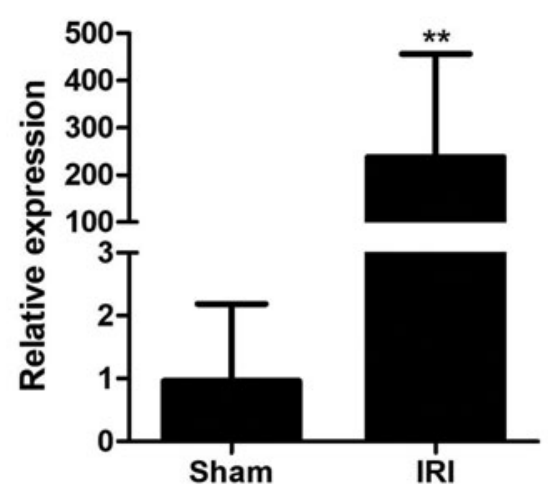

C

Sham

MMP-10

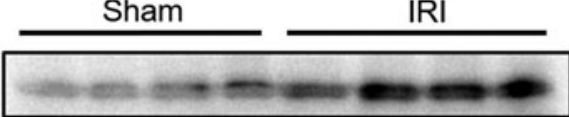

$\beta$-actin

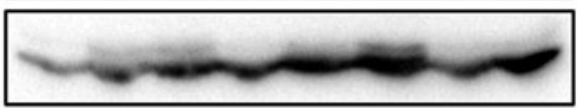

e

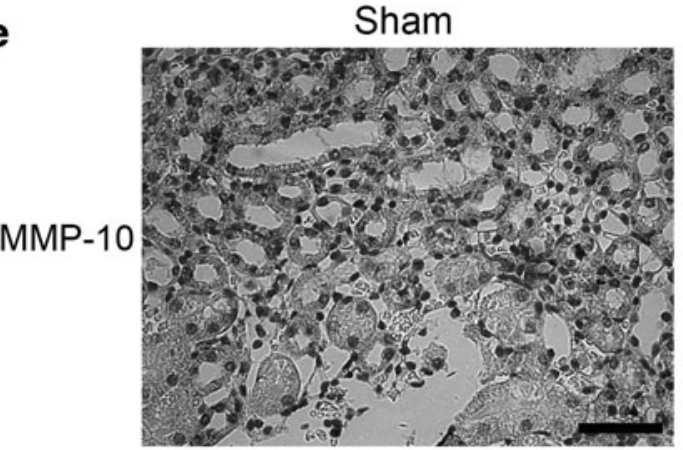

$54 \mathrm{KD}$

$43 \mathrm{KD}$

b

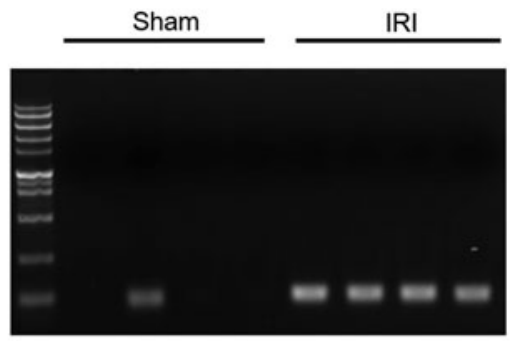

GAPDH
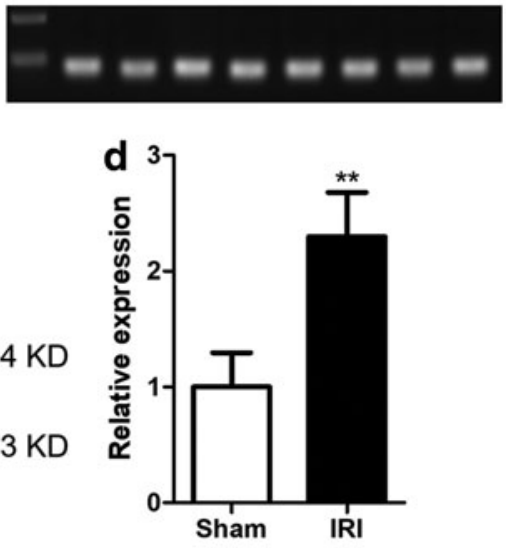

IRI

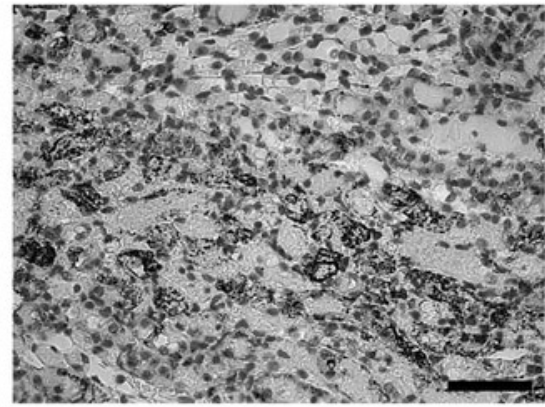

FIG. 3. $M M P-10$ is induced by renal IRI. $(\mathbf{a}, \mathbf{b})$ The expression of $M M P-10$ was measured using a realtime qPCR assay (a).

$G A P D H$ was used as an internal control. Data are expressed as mean \pm standard deviation and $* * p<0.01$ versus the sham-operated group ( $n=4$ per group). The qPCR of products were separated by agarose electrophoresis and observed through an ultraviolet imager (b). (c, d) The protein levels of renal MMP-10 was detected using western blotting (c). The expression intensity for each band was scanned and normalized to the $\beta$-actin intensity in each lane (d). (e) Immunohistochemistry staining was performed to localize the MMP-10 in kidney tissues. Scale bars: $50 \mu \mathrm{m}$. MMP, matrix metalloproteinases. 

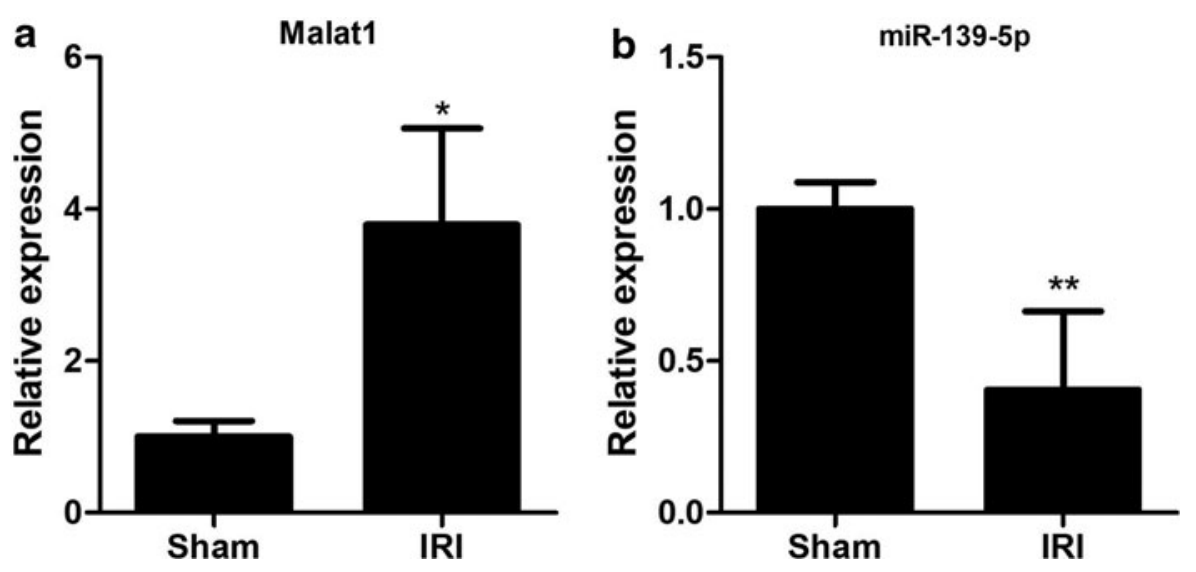

FIG. 4. The expression of lncRNA Malat1 and $m i R-139-5 p$ in kidneys of IRI. (a) The expression of lncRNA Malat1 in kidneys was detected using real-time qPCR assay. GAPDH was used as an internal control. (b) The relative expression of $m i R-139-5 p$ was detected using a "stem-loop" qPCR method. U6 was used as an internal control. Data are expressed as mean \pm standard deviation and normalized using a $2^{-\Delta \Delta \mathrm{Ct}}$ assay. $* p<0.05$ and $* * p<0.01$ versus the sham-operated mice $(n=4$ per group). lncRNA, long noncoding RNA.

strongly stained in the injury area of the kidney, but weakly stained in kidneys of sham-operated mice (Fig. 3e). Our data suggested that MMP-10 might be a potential molecular candidate participating in renal IRI.

\section{The expression of Malat1 and miR-139-5p in renal IRI}

Noncoding RNAs, including miRNAs, lncRNAs, and circular RNAs (circRNAs), play important roles in many biological processes. Our data of microarray screened that the lncRNA Malatl was upregulated in IRI. The real-time qPCR verified that Malat1 in kidneys of IRI mice was increased to 3.79-fold compared with the sham-operated mice (Fig. 4a). In addition, we detected the expression of $m i R-139-5 p$, which is not included in the microarray data but closely associated with cell proliferation. IRI treatment repressed the renal miR-139$5 p$ expression to $40.4 \%$ of the sham-operated mice (Fig. $4 \mathrm{~b}$ ). These data demonstrated that both noncoding RNAs Malat1 and $m i R-139-5 p$ were involved in IRI.

\section{Discussion}

Renal IRI is a main risk factor for the occurrence of DGF and PGN in kidney transplantation. IRI is a multistep pathological process and is currently not fully clarified. Therefore, it lacks an ideal biomarker for the diagnosis and treatment. Recipients undergo IRI of the graft frequently requires a redialysis, causing heavy burden both physically and mentally. Therefore, identification of novel molecular biomarkers is important for the diagnosis and treatment of DGF and PGN. Previously, we screened differentially expressed genes in experimental IRI models through a microarray assay. In the present study, we used these data to perform enrichment analysis and identified that $S P R R 2 F, S P R R 1 A, M M P-10$, and Malatlwere significantly altered in our experimental models, indicating that these genes may be involved in renal IRI.

$S P R R 2 F$ and SPRR1A belong to the small proline-rich protein family, which are both important markers of keratinocyte terminal differentiation (Sark et al., 1998; Chen et al., 2010). SPRR2F is highly expressed in uterus and ovary (Song et al., 1999; Cabral et al., 2001) and briefly expressed during nephrogenesis (Schwab et al., 2003). We observed that $S P R R 2 F$ was seldom expressed in normal kidney tissues, but could be strongly induced by IRI treatment. The function of $S P R R 2 F$ remains far from understanding. In contrast, SPRRIA, another member of SPRR family, is well profiled and is known to be associated with many diseases, such as breast cancer and diffuse large B cell lymphoma (Zhang et al., 2014; Chen et al., 2015). SPRRIA is a gene related with tissue regeneration (Gaudet et al., 2016; Gey et al., 2016; Danaher et al., 2018) and can be controlled by ATF3 (Danaher et al., 2018). In the present study, we found an increased expression of SPRRIA in kidneys of IRI-treated mice. Therefore, we speculate that $S P R R 2 F$ and SPRR1A are biomarkers of kidney repair and regeneration after IRI, which may reflect the recovery of the kidney function. However, the detailed role of $S P R R 2 F$ and $S P R R 1 A$ in renal IRI remains to be investigated.

Most of the MMPs are secreted enzymes mediating ECM degradation. MMP-10 (also known as stromelysin 2, SL-2) belongs to the stromelysins subgroup of $M M P s$, catalyzing substrates include collagen IV, laminin, fibronectin, and elastin. MMP-10 was reported to be associated with atherosclerosis (Purroy et al., 2018), intracerebral hemorrhage (Howe et al., 2018), pulmonary hypertension (Avouac et al., 2017), and cancers (Miyata et al., 2007; Du et al., 2014; Kadeh et al., 2016; Juchniewicz et al., 2017). Recently, Guvercin et al. reported that the increase of serum $M M P-10$ is related to acute kidney injury in nondiabetic geriatric patients (Guvercin et al., 2018). Our data demonstrated that the expression of $M M P-10$ in normal kidney tissues is very weak, but could be strongly activated by IRI treatment, supporting that $M M P-10$ is a potential candidate involved in renal IRI. Interestingly, we observed that the expression of $M M P-10$ at mRNA level was not paralleled with its protein level in normal kidney tissues. Because mature form of MMP-10 enzyme can be secreted into the circulation, we postulate that the circulatory MMP-10 in the plasma may be recruited to the kidney, causing the present of MMP-10 protein in the kidney tissues. Due to the stromelysin activity, the upregulation of $M M P-10$ in tissues results in the degradation and remodeling of the ECM (Avouac et al., 2017), further causing intestinal fibrosis and thus hindering the 
recovery of kidney function (Sokai et al., 2015). Our data indeed showed a significant accumulation of MMP-10 in the injured area of the kidney, indicating that tissue MMP-10 may be a potential biomarker to reflect the severity of tissue injury. We speculate that MMP-10 may be a potential therapeutic target for acute kidney injury.

From the data of the differentially expressed genes, we found that lncRNA Malat1 was upregulated in kidneys of IRItreated mice, which has been reported from other groups (Kölling et al., 2018; Tian et al., 2018). However, these reports remain controversy. Tian et al. reported that the increase of Malat1 inhibits hypoxia-induced inflammation (Tian et al., 2018), whereas Kölling, et al. speculated Malat1 as dispensable (Kölling et al., 2018). Our data support that Malat1 participates in IRI, but its role remains to be clarified. In addition to the high throughput data, we identified that $m i R$ $139-5 p$ was decreased in renal IRI. $m i R-139-5 p$ was reported to inhibit tumorigenesis and reverses drug resistance in many cancers (Wang et al., 2017; Jiang et al., 2018; Zhang et al., 2018). Targets of $m i R-139-5 p$, such as c-Jun (Jiang et al., 2018; Su et al., 2018), PI3K/Akt (Maoa et al., 2015; Catanzaro et al., 2018), IGF-1/IGF-1R (Xu et al., 2015), VEGF, and Wnt/ $\beta$-catenin (Long et al., 2017; Xie and Shen, 2018), are potent stimulators of cell proliferation, some of which are important pathways promoting injury repair (Zhou et al., 2016; Guise and Chade, 2018; Xie et al., 2018). Therefore, we postulate that the downregulation of $m i R-139-5 p$ may reflect an activation of cell proliferation, promoting the repair and regeneration of injured kidney.

Taken together, our data indicated that the expression of SPRR2F, SPRR1A, MMP-10, Malat1, and miR-139-5p in kidney was altered by IRI, which may serve as potential biomarkers for renal IRI. These findings may also provide evidences for exploring novel targets for the prevention and treatment of DGF and PGN after kidney transplantation.

\section{Acknowledgments}

This study was supported by the National Natural Science Foundation of China (No. 81602214 and 81500236) and Beijing Municipal Administration of Hospitals Clinical Medicine Development of Special Funding Support (ZYLX201604).

\section{Disclosure Statement}

No competing financial interests exist.

\section{Supplementary Material}

Supplementary Table S1

Supplementary Table S2

\section{References}

Avouac, J., Guignabert, C., Hoffmann-Vold, A.M., Ruiz, B., Dorfmuller, P., Pezet, S., et al. (2017). Role of stromelysin 2 (matrix metalloproteinase 10) as a novel mediator of vascular remodeling underlying pulmonary hypertension associated with systemic sclerosis. Arthritis Rheumatol 69, 2209-2221.

Cabral, A., Voskamp, P., Cleton-Jansen, A.M., South, A., Nizetic, D., and Backendorf, C. (2001). Structural organization and regulation of the small proline-rich family of cornified envelope precursors suggest a role in adaptive barrier function. J Biol Chem 276, 19231-19237.
Catanzaro, G., Besharat, Z.M., Miele, E., Chiacchiarini, M., Po, A., Carai, A., et al. (2018). The miR-139-5p regulates proliferation of supratentorial paediatric low-grade gliomas by targeting the PI3K/AKT/mTORC1 signalling. Neuropathol Appl Neurobiol 44, 687-706.

Chen, G., Li, G., Luo, M., Wei, X., Wang, D., Zhang, H., et al. (2015). Clinical significance of SPRR1A expression in progesterone receptor-positive breast cancer. Tumour Biol 36, 2601-2605.

Chen, J., Chen, Y., Capizzi, S., Yang, M., Deng, L., Bledsoe, S.B., et al. (2010). 2,8-dihydroxyadenine nephrolithiasis induces developmental stage-specific alterations in gene expression in mouse kidney. Urology 75, 914-922.

Danaher, R.J., Zhang, L., Donley, C.J., Laungani, N.A., Hui, S.E., Miller, C.S., et al. (2018). Histone deacetylase inhibitors prevent persistent hypersensitivity in an orofacial neuropathic pain model. Mol Pain 14, 1744806918796763.

Danobeitia, J.S., Ziemelis, M., Ma, X., Zitur, L.J., Zens, T., Chlebeck, P.J., et al. (2017). Complement inhibition attenuates acute kidney injury after ischemia-reperfusion and limits progression to renal fibrosis in mice. PLoS One 12, e0183701.

Du, X., Lin, B.C., Wang, Q.R., Li, H., Ingalla, E., Tien, J., et al. (2014). MMP-1 and Pro-MMP-10 as potential urinary pharmacodynamic biomarkers of FGFR3-targeted therapy in patients with bladder cancer. Clin Cancer Res 20, 6324-6335.

Gaudet, A.D., Mandrekar-Colucci, S., Hall, J.C., Sweet, D.R., Schmitt, P.J., Xu, X., et al. (2016). miR-155 deletion in mice overcomes neuron-intrinsic and neuron-extrinsic barriers to spinal cord repair. J Neurosci 36, 8516-8532.

Gey, M., Wanner, R., Schilling, C., Pedro, M.T., Sinske, D., and Knoll, B. (2016). Atf3 mutant mice show reduced axon regeneration and impaired regeneration-associated gene induction after peripheral nerve injury. Open Biol 6, 160091.

Gu, L., Tao, Y., Chen, C., Ye, Y., Xiong, X., and Sun, Y. (2018). Initiation of the inflammatory response after renal ischemia/reperfusion injury during renal transplantation. Int Urol Nephrol 50, 2027-2035.

Guise, E., and Chade, A.R. (2018). VEGF therapy for the kidney: emerging strategies. Am J Physiol Renal Physiol 315, F747-F751.

Guvercin, G., Karakus, V., Aksit, M., Dere, Y., Aktar, M., Alpay, H., et al. (2018). Matrix metalloproteinase-9, 10, and stress hyperglycaemia in acute kidney injury. Eur J Clin Invest $\mathbf{4 8}$, e12963.

Hesketh, E.E., Czopek, A., Clay, M., Borthwick, G., Ferenbach, D., Kluth, D., et al. (2014). Renal ischaemia reperfusion injury: a mouse model of injury and regeneration. $\mathrm{J}$ Vis Exp. DOI: $10.3791 / 51816$.

Howe, M.D., Zhu, L., Sansing, L.H., Gonzales, N.R., McCullough, L.D., and Edwards, N.J. (2018). Serum markers of blood-brain barrier remodeling and fibrosis as predictors of etiology and clinicoradiologic outcome in intracerebral hemorrhage. Front Neurol 9, 746.

$\mathrm{Hu}, \mathrm{X} ., \mathrm{Su}$, M., Lin, J., Zhang, L., Sun, W., Zhang, J., et al. (2019). Corin is downregulated in renal ischemia/reperfusion injury and is associated with delayed graft function after kidney transplantation. Dis Markers 2019, https://doi.org/10.1155/ 2019/9429323

Jain, S., Bicknell, G.R., and Nicholson, M.L. (2000). Molecular changes in extracellular matrix turnover after renal ischaemiareperfusion injury. Br J Surg 87, 1188-1192.

Jiang, Y., Jiang, J., Jia, H., Qiao, Z., and Zhang, J. (2018). Recovery of miR-139-5p in ovarian cancer reverses cisplatin resistance by targeting c-Jun. Cell Physiol Biochem 51, 129-141. 
Juchniewicz, A., Kowalczuk, O., Milewski, R., Laudanski, W., Dziegielewski, P., Kozlowski, M., et al. (2017). MMP-10, MMP-7, TIMP-1 and TIMP-2 mRNA expression in esophageal cancer. Acta Biochim Pol 64, 295-299.

Kadeh, H., Saravani, S., Heydari, F., and Shahraki, S. (2016). Differential immunohistochemical expression of matrix metalloproteinase-10 (MMP-10) in non-melanoma skin cancers of the head and neck. Pathol Res Pract 212, 867871.

Kim, D.J., Kang, J.M., Park, S.H., Kwon, H.K., Song, S.J., Moon, H., et al. (2017). Diabetes aggravates post-ischaemic renal fibrosis through persistent activation of TGF-beta1 and Shh signalling. Sci Rep 7, 16782.

Kölling, M., Genschel, C., Kaucsar, T., Hubner, A., Rong, S., Schmitt, R., et al. (2018). Hypoxia-induced long non-coding RNA Malat1 is dispensable for renal ischemia/reperfusioninjury. Sci Rep 8, 3438.

Liu, J.H., He, L., Zou, Z.M., Ding, Z.C., Zhang, X., Wang, H., et al. (2018). A novel inhibitor of homodimerization targeting MyD88 ameliorates renal interstitial fibrosis by counteracting TGF-beta1-induced emt in vivo and in vitro. Kidney Blood Press Res 43, 1677-1687.

Long, H., Sun, B., Cheng, L., Zhao, S., Zhu, Y., Zhao, R., et al. (2017). miR-139-5p represses BMSC osteogenesis via targeting wnt/beta-catenin signaling pathway. DNA Cell Biol 36, 715-724.

Maoa, R., Zou, F., Yang, L., Lin, S., Li, Y., Ma, M., et al. (2015). The loss of MiR-139-5p promotes colitis-associated tumorigenesis by mediating PI3K/AKT/Wnt signaling. Int $\mathrm{J}$ Biochem Cell Biol 69, 153-161.

Miyata, Y., Iwata, T., Maruta, S., Kanda, S., Nishikido, M., Koga, S., et al. (2007). Expression of matrix metalloproteinase10 in renal cell carcinoma and its prognostic role. Eur Urol 52, 791-797.

Molls, R.R., Savransky, V., Liu, M., Bevans, S., Mehta, T., Tuder, R.M., et al. (2006). Keratinocyte-derived chemokine is an early biomarker of ischemic acute kidney injury. Am J Physiol Renal Physiol 290, F1187-F1193.

Pressly, J.D., and Park, F. (2017). DNA repair in ischemic acute kidney injury. Am J Physiol Renal Physiol 312, F551-F555.

Purroy, A., Roncal, C., Orbe, J., Meilhac, O., Belzunce, M., Zalba, G., et al. (2018). Matrix metalloproteinase-10 deficiency delays atherosclerosis progression and plaque calcification. Atherosclerosis 278, 124-134.

Sanchez-Nino, M.D., Sanz, A.B., and Ortiz, A. (2016). Chronicity following ischaemia-reperfusion injury depends on tubular-macrophage crosstalk involving two tubular cellderived CSF-1R activators: CSF-1 and IL-34. Nephrol Dial Transplant 31, 1409-1416.

Sark, M.W., Fischer, D.F., de Meijer, E., van de Putte, P., and Backendorf, C. (1998). AP-1 and ets transcription factors regulate the expression of the human SPRR1A keratinocyte terminal differentiation marker. J Biol Chem 273, 24683-24692.

Schwab, K., Patterson, L.T., Aronow, B.J., Luckas, R., Liang, H.C., and Potter, S.S. (2003). A catalogue of gene expression in the developing kidney. Kidney Int 64, 1588-1604.

Situmorang, G.R., and Sheerin, N.S. (2018). Ischaemia reperfusion injury: mechanisms of progression to chronic graft dysfunction. Pediatr Nephrol [Epub ahead of print]; DOI: 10.1007/s00467-018-3940-4.
Sokai, A., Handa, T., Tanizawa, K., Oga, T., Uno, K., Tsuruyama, T., et al. (2015). Matrix metalloproteinase-10: a novel biomarker for idiopathic pulmonary fibrosis. Respir Res 16, 120.

Song, H.J., Poy, G., Darwiche, N., Lichti, U., Kuroki, T., Steinert, P.M., et al. (1999). Mouse Sprr2 genes: a clustered family of genes showing differential expression in epithelial tissues. Genomics 55, 28-42.

Su, M., Wang, S.Y., Qiu, W., Li, J.H., Hui, R.T., Song, L., et al. (2018). miR-139-5p inhibits isoproterenol-induced cardiac hypertrophy by targetting c-Jun. Biosci Rep 38, BSR20171430.

Tian, H., Wu, M., Zhou, P., Huang, C., Ye, C., and Wang, L. (2018). The long non-coding RNA MALAT1 is increased in renal ischemia-reperfusion injury and inhibits hypoxiainduced inflammation. Ren Fail 40, 527-533.

Wang, K., Jin, J., Ma, T., and Zhai, H. (2017). MiR-139-5p inhibits the tumorigenesis and progression of oral squamous carcinoma cells by targeting HOXA9. J Cell Mol Med 21, 3730-3740.

Xie, H., Wang, Y., Zhang, H., Fan, Q., Dai, D., Zhuang, L., et al. (2018). Tubular epithelial C1orf54 mediates protection and recovery from acute kidney injury. J Cell Mol Med 16, 4985-4996.

Xie, Y., and Shen, G. (2018). MicroRNA-139-5p elevates skeletal myogenic differentiation of human adult dental pulp stem cells through Wnt/beta-catenin signaling pathway. Exp Ther Med 16, 2835-2842.

Xu, W., Hang, M., Yuan, C.Y., Wu, F.L., Chen, S.B., and Xue, K. (2015). MicroRNA-139-5p inhibits cell proliferation and invasion by targeting insulin-like growth factor 1 receptor in human non-small cell lung cancer. Int J Clin Exp Pathol 8, 3864-3870.

Zhang, H., Gao, J., Zhao, Z., Li, M., and Liu, C. (2014). Clinical implications of SPRR1A expression in diffuse large B-cell lymphomas: a prospective, observational study. BMC Cancer 14, 333.

Zhang, R., Tang, P., Wang, F., Xing, Y., Jiang, Z., Chen, S., et al. (2018). Tumor suppressor miR-139-5p targets Tspan3 and regulates the progression of acute myeloid leukemia through the PI3K/Akt pathway. J Cell Biochem [Epub ahead of print]; DOI: $10.1002 /$ jcb.27728.

Zhou, D., Tan, R.J., Fu, H., and Liu, Y. (2016). Wnt/ $\beta$-catenin signaling in kidney injury and repair: a double-edged sword. Lab Invest 96, 156-167.

Address correspondence to: Wei Qiu, MD, PhD

Department of Urology

Beijing Friendship Hospital

Capital Medical University

No. 95 Yongan Road

Xicheng District

Beijing 100050

People's Republic of China

E-mail: qiuwei618@163.com

Received for publication November 25, 2018; received in revised form December 10, 2018; accepted December 17, 2018. 